\title{
Weighted Centroid Range Free Localization Algorithm based on IOT
}

\author{
Neha Sharma \\ Dept. of Computer Sc. \& Engg \\ ITM University \\ Gurgaon, India
}

\author{
Gazal Chawla \\ Dept. of Computer Sc. \&Engg \\ ITM University \\ Gurgaon, India
}

\author{
Mehak Khurana \\ Dept. of Computer Sc.\& Engg \\ ITM University \\ Gurgaon, India
}

\begin{abstract}
Today, IOT (Internet of Things or Internet of Objects) is popular upcoming topic in Wireless network and in Wireless Network. Localization has become a crucial factor for various monitoring applications such as search, rescue, disaster relief, target tracking etc. Various WSN localization technologies are designed for calculating location of the unknown node. IOT is a latest approach where it combines many technologies, such as network technology, communication technology, database technology (Internet, Bluetooth, infrared, Wi-Fi, GPRS, 3G, $\mathrm{SQL}$, etc) to provide location based service which enables different ways to obtain the location information of various objects. In this paper, we focus on to achieve higher localization accuracy based on services in which we deploy sensing devices to calculate the information of sensor nodes and its surrounding nodes where it requires less hardware and less implementation, To improve the localization accuracy, we propose a Weighted Centroid based on IOT mechanism capable to give accurate calculation between Anchor node and Unknown node.
\end{abstract}

\section{Keywords}

Wireless Sensor Network, Internet of Things, Weighted Centroid, localization.

\section{INTRODUCTION}

Wireless Sensor Network (WSN) is spread networks consisting a huge number of sensor nodes, which are distributed in the various areas include target tracking, disaster relief and smart environments etc. In these WSN application, position of nodes is one of the crucial issue. There are various localization techniques to provide location information of each deployed sensor node in a sensor network. The goal in WSN is to obtain maximum localization accuracy with minimum computations. The position of the nodes can be known if these nodes deployed are Global Positioning System (GPS) [1] [2] equipped. These nodes whose position is well known are called Anchor nodes [1] (GPS equipped) but GPS has some limitations such as power consumption and large cost. In a wireless sensor network all the nodes cannot be Anchor node because of its limitations. So some GPS equipped nodes are deployed to infer the position of rest of the nodes. These nodes whose positions are not known are called Unknown nodes.
Localization can be divided into two methods: Range Based and Range Free. Range based method [3] [4] localizes the Unknown node by calculating the exact distance or angle between anchor nodes and unknown nodes and provides high accuracy but it requires extra hardware. This method includes TOA [3] [5] (Time of arrival), TDOA [4] (Time difference of arrival), AOA [4] [5] (angle of arrival) and RSSI [3] [5] (Received Signal Strength Indicator). The other method is Range free [4] which does not calculates actual distance between anchor nodes and unknown nodes and consumes less energy with less cost as it does not require extra hardware, but it does not provide high accuracy. Range Free includes APIT algorithm [5], amorphous algorithm [6], centroid algorithm [7][8][9], Dv-hop algorithm [10][11][12][13]. Localization accuracy of Range Based is higher than that of range free method but range free is easily implemented and is suitable for many applications such as monitoring applications.

There are two main Range Free algorithms: Centroid and DVHop algorithm .In DV-Hop algorithm [10][11][12] position of the Unknown node is calculated on the basis of hop count. So to localize a node multi-hops calculation ranging Unknown node and anchor node are performed. This multi-hopping between the nodes consumes large energy. So our main focus is on energy efficient algorithm that is Centroid algorithm where the distance is calculated between the nodes and the centroid is calculated.

IOT [14][15] is popular upcoming topic and rapidly gaining grounds in field of wireless communication. IOT is a latest approach where it combines many technologies, such as Internet, Bluetooth, infrared, Wi-Fi, GPRS, 3G, etc to provide location based service which enables different ways to obtain the location information of various objects. IOT plays an important role in Network field. The term IOT is identified by a unique address, unique addressing scheme which helps the objects to interact with variety of other objects placed in a field. Any object including computers, sensors, RFID tags or mobile phones will be able to dynamically join the network. IOT is the latest technology which provides based service which enables to gather information of sensor nodes and surrounding nodes automatically and send that gathered information where it is required. In this paper, centroid algorithm based on IOT is proposed. This paper is analyzed which shows that this algorithm provides better accuracy and less estimation error. 


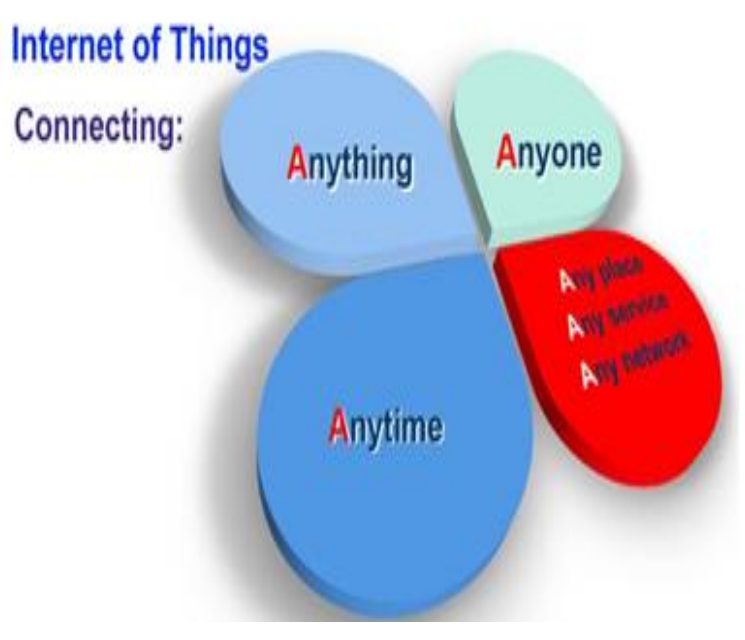

Fig 1: Internet of Things

\section{IOT BENIFITS}

The IOT [16] could allow things and people to be connected Anyplace, Anytime with Anything and Anyone using Any Network and Any Service. IOT collects the surrounding information automatically and sends that information wherever it is required. In IOT password can be maintained which provide security. IOT stores all the information for indefinite time in its database, and that information can be retrieved whenever it is required. Heterogeneous technologies can also exist in IOT. The possible application domains in IOT are industry, society, environment etc. this is shown in figure 2. The various applications under this domain are Manufacturing, Product Lifecycle Management, People and Goods Transportations, Recycling, Intelligent buildings, Telecommunications etc.

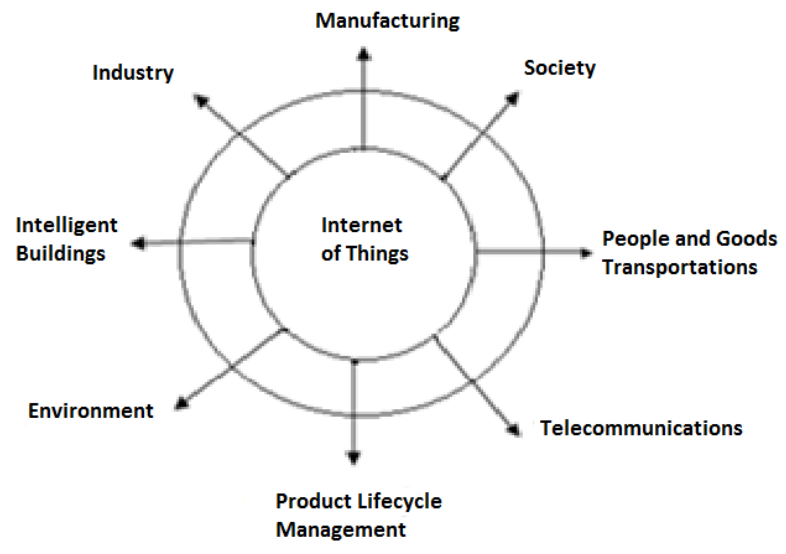

Fig 2: IOT Application Domain

\section{LOCALIZATION FACTORS}

In this method [14], some factors are taken into consideration so to get accurate results on time with quality and low energy consumption.

Energy Efficiency: Energy conservation and consumption is a key issue for sensors because sensors have low battery life. So we require a solution where the main objective is to conserve energy to level zero. Weighted Centroid uses only single hop technique to send message between Anchor nodes and Unknown nodes rather than multi-hops because single hop consumes less energy and less computation complexity. Due to which Weighted Centroid method is better as compared to other range free algorithms.

Accuracy: The main goal is to ensure the high accuracy in the proposed algorithm. We use RSSI to calculate the distance between Anchor nodes and Unknown nodes because RSSI provides high accuracy.

Location awareness: For location awareness IOT helps to reduce latency in information as it automatically collects the information so the information is received on time due to which quality of service improves.

Power system stability: IOT's application significantly improves power system and stability.

Interoperability: Interoperability is to exchange information between two or more networks which is a major issue in wireless sensor network. We require such a method that allows interoperability. For solving the interoperability problem IOT devices are used which provides exchange of data.

Security: Security is an important issue in Wireless Sensor Network. in a network byzantine sensors can be introduced by the intruders which can damage the inward security. So to maintain the security a method should be adopted for byzantine sensor identification. IOT happens only when there is strong security.

\section{PROPOSED ALGORITHM}

Three Steps to calculate the Weighted Centroid considering all the above mentioned factors:

Step 1: Anchor node senses the anchor message to all the nodes within communication range. Here, IOT helps to send the information on time due to which quality of service is improved. All the nodes (Anchor nodes and Unknown nodes) receive the information from Anchor nodes and each Unknown nodes keeps only that information which includes single hop due to which it consumes less energy.

Step 2: Now polygon is formed by all Anchor nodes. Anchor nodes calculate the distance to Unknown nodes using RSSI. RSSI provides accurate results due to its ease of implementation and estimation.

Step 3: Centroid is calculated using weighted Centroid formula
$X_{e s t}=\frac{\sum_{i=1}^{n} w_{i} x_{i}}{\sum_{i=1}^{n} w_{i}}=\frac{w_{1} x_{1}+w_{2} x_{2}+\ldots \ldots \ldots \ldots \ldots+w_{n} x_{n}}{w_{1}+w_{2}+\ldots \ldots \ldots \ldots \ldots . .+w_{n}}$ 
$Y_{\text {est }}=\frac{\sum_{i=1}^{n} w_{i} y_{i}}{\sum_{i=1}^{n} w_{i}}=\frac{w_{1} y_{1}+w_{2} y_{2}+\ldots \ldots \ldots \ldots \ldots+w_{n} y_{n}}{w_{1}+w_{2}+\ldots \ldots \ldots \ldots \ldots+w_{n}}$

(2)

$($ Xest, Yest $)=$ estimated co-ordinates of unknown node

$\mathrm{n}=$ number of anchor nodes

$\mathrm{w}_{\mathrm{i}=}$ represent the weighted factor.

$\left(\mathrm{x}_{\mathrm{i},} \mathrm{y}_{\mathrm{i}}\right)=$ are co-ordinates of anchor nodes.

And the location coordinates is broadcasted to all the nodes and that information of target node is send to the server where it is required.

The Table 2 represents the estimated errors for Unknown nodes and the symbols are presented of figure 3 .

Table 2: Symbol Representation

\begin{tabular}{|l|l|}
\hline- & $\begin{array}{l}\text { Solid blue dots indicate anchor } \\
\text { nodes. }\end{array}$ \\
\hline$\circ$ & $\begin{array}{l}\text { No filled circle indicates } \\
\text { unknown nodes. }\end{array}$ \\
\hline$*$ & $\begin{array}{l}\text { Red colored star indicates actual } \\
\text { position of error }\end{array}$ \\
\hline- & $\begin{array}{l}\text { Red colored line indicates the } \\
\text { difference between the actual } \\
\text { position of error and estimated } \\
\text { location. }\end{array}$ \\
\hline
\end{tabular}

Now this below figure 3 shows that 12 Anchor nodes are deployed grid wise uniformly and 15 Unknown nodes are deployed randomly and communication range of each node is set as 30. Weighted Centroid is calculated using equation 1 and 2 and the estimated error is calculated. This figure shows that the estimated less error.

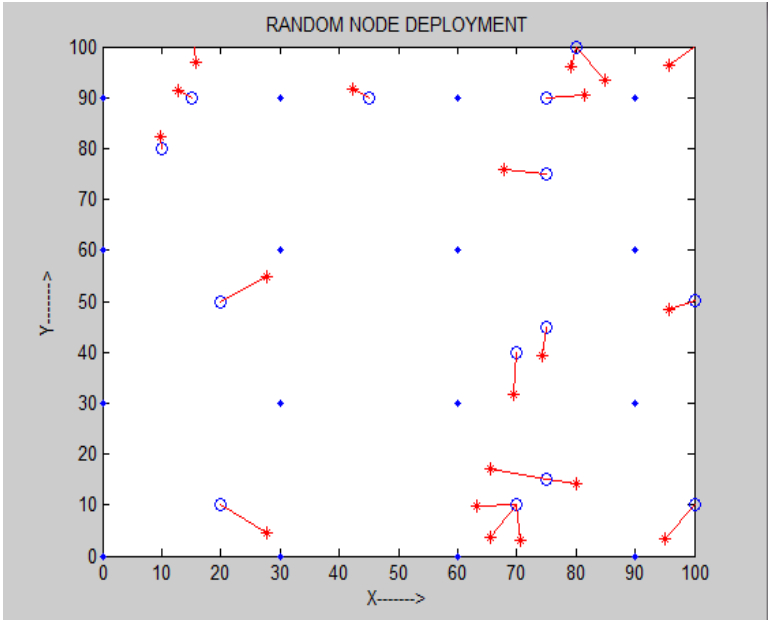

Fig 3: shows estimated error of unknown node

\section{ISSUES FOR IOT}

There are some technical issues for IOT and these issues are discussed below:

1. IOT generates information traffic in a current internet, as it collects the information automatically and sends to the destination.

2. In IOT password length is too short to provide high security.

3. The private information is automatically collected without informing the conserved person which can be a threat to a security.

4. The cost of storage of information is high as it stores all the information for infinite period

5. In IOT, existing transport protocol does not support.

6. Scalability to the heterogeneous technologies in IOT can represent crucial problems.

\section{COMPARISON BETWEEN CENTROID AND PROPOSED ALGORITHM}

\begin{tabular}{|c|c|c|c|c|c|c|c|}
\hline \multirow{2}{*}{$\begin{array}{l}\text { LOCALIZATION } \\
\text { ALGORITHM }\end{array}$} & \multicolumn{7}{|l|}{ Factors } \\
\hline & $\begin{array}{l}\text { ENERGY } \\
\text { EFFICIET }\end{array}$ & COMMUNICATION & $\begin{array}{l}\text { QUALITY } \\
\text { OF } \\
\text { SERVICE }\end{array}$ & $\begin{array}{l}\text { DISTANCE } \\
\text { CALCULATION }\end{array}$ & $\begin{array}{l}\text { ESTIMATED } \\
\text { ERROR }\end{array}$ & ACCURACY & $\begin{array}{l}\text { INTERO } \\
\text { PERABILITY }\end{array}$ \\
\hline $\begin{array}{l}\text { CENTROID } \\
\text { ALGORITHM }\end{array}$ & YES & NO & LESS & $\begin{array}{l}\text { DISTANCE } \\
\text { FORMULA }\end{array}$ & LARGE & LOW & NO \\
\hline $\begin{array}{l}\text { PROPOSED } \\
\text { ALGORITHM }\end{array}$ & $\begin{array}{l}\text { YES, } \\
\text { SINGLE } \\
\text { HOP }\end{array}$ & $\begin{array}{l}\text { YES, BECAUSE IT } \\
\text { USES IOT }\end{array}$ & $\begin{array}{l}\text { LARGE, } \\
\text { BECAUSE } \\
\text { OF IOT }\end{array}$ & USING RSSI & LESS & HIGH & YES \\
\hline
\end{tabular}




\section{CONCLUSION}

Localization is an important issue in Wireless Sensor Network. Proposed algorithm provides the location coordinates of the node with accuracy. In this proposed algorithm IOT places a major role to send the information on time to all nodes in the network due to which quality of service is improved. This proposed algorithm is a single hop algorithm due to which energy consumption and computation complexity is less. it also maintains security from byzantine sensors. But there are some issues for IOT that are addressed above which need to be overcome.

\section{REFERENCES}

[1] Jasmin Desai, UfTureli, "Evaluating Performance Of Various Localization Algorithms In Wireless Sensor Networks," The $18^{\text {th }}$ Annual International Symposium on Personal, Indoor and Mobile Radio Communication (PIMRC' 07), 2007.

[2] M. Abdelhadi, M. Anan, M. Ayyash, "Efficient Artifical Intelligent-based Localization Algorithm for Wireless Sensor Networks," Cyber Journals: Multidisciplinary Journals in Science and Technology, Journal of Selected Areas in Telecommunications (JSAT), vol. 3, 2013.

[3] Amitangshu.Pal, "Localization Algorithms in Wireless Sensor Networks: Current Approaches and Future Challenges," Networks Protocols and Algorithms, ISSN 1943-3581, Vol. 2, No. 1, 2010

[4] ShayonSamanta, Prof. Punesh U. Tembhare, Prof. CharanR.Pote, "A Survey on 3d Localization in Wireless Sensor Networks," International Journal Of Computational Engineering Research (ijceronline.com), Vol. 3, Issue. 1, pp.90-94, January 2013.

[5] Vishal Garg, Mukul Jjamb, "A Review of Wireless Sensor Network on Localization Techniques," International Journal of Engineering Trends and Technology (IJETT)Volume4Isssue4-April 2013.

[6] Tian He, Chengdu Huang, Brian M. Blum, John A. Stankovic, TarekAbdelzaher, "Range-Free Localization Schemes for Large Scale Sensor Network," MobiCom '03, September 14-19, 2003, San Diego, California, USA.
[7] R. Behnke, D.Timmermann, "AWCL:Adaptive Weighted Centroid Localization as an Efficient Improvement of Coarse Grained Localization," PROCEEDINGS OF THE $5^{\mathrm{TH}}$ WORKSHOP ON POSITIONING , NAVIGATION AND COMMUNICATION , pp. 243-250, 2008.

[8] Bingjiao Zhang, MinningJi, Lianhai Shan, "A Weighted Centroid Localization Algorithm Based on DV-Hop for Wireless Sensor Network," 2012.

[9] Yu HU, Weizhao YAO, "Weighted Centroid Localization Algorithm Using Linear Regression for Wireless Sensor Networks," Journal of Computational information Systems 7: 9 (2011) 3268-3274, September 2011.

[10] "Localization of Nodes in Wireless Sensor Networks by MDV-Hop Algorithm," ARPN Journal of Systems and a Software, Vol. 2, NO. 5, MAY 2012.

[11] Shrawan Kumar and D.K. Lobiyal, "Improvement over DV-Hop Localization Algorithm for Wireless Sensor Networks," World Academy of Sciences, Engineering and Technology, pp. 282-292. 2013.

[12] Weihua Pan, Xiaodan Liu, "Wireless Sensor Networks based on DV-Hop Localization Algorithm" Fourth International Conference on Computational and Information Sciences, pp.1073-1075, 2012.

[13] Wenqi Yu, Hao Li, “An Improved DV-Hop Localization Method in Wireless Sensor Networks," pp.199-202, 2012.

[14] Liang Hu, Zhengyu Zhang, Feng Wang, and Kuo Zhao, "Optimization of the Deployment of Temperature Nodes Based on Linear Programming in the Internet of Things" Tsinghua Science and Technology, pp.250-258, Volume 18 ,Number3, June2013.

[15] "INTERNET OF THINGS STATEGIC RESEARCH ROAPMAP", $15^{\text {th }}$ September, 2009.

[16] Liang Liu, Xi Zhang, Senior Member, IEEE, and Huadong Ma, Member, IEEE, "Percolation Theory-Based Exposure-Path Prevention for Wireless Sensor Networks Coverage in Internet of Things" IEEE Sensors Journal, Vol. 13, no. 10, October2013. 